LA-UR- 95-1683

Title:

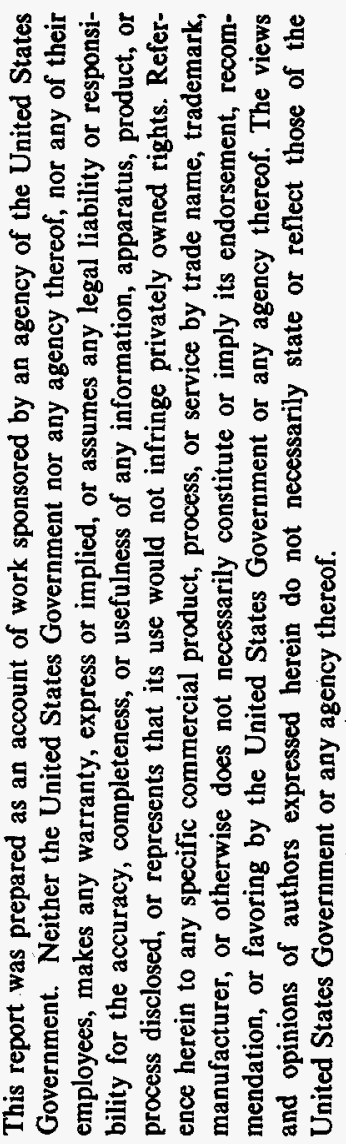

Author(s):

Submitted to:
TIME STRUCTURE IN THE MARCH 5TH 1979 EVENT

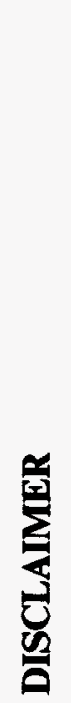

\section{Los Alamos} NATIONAL LABORATORY
E. E. Fenimore

High Velocity Neutron Stars and Gamma Ray Bursts San Diego, CA

March 15-17, 1995

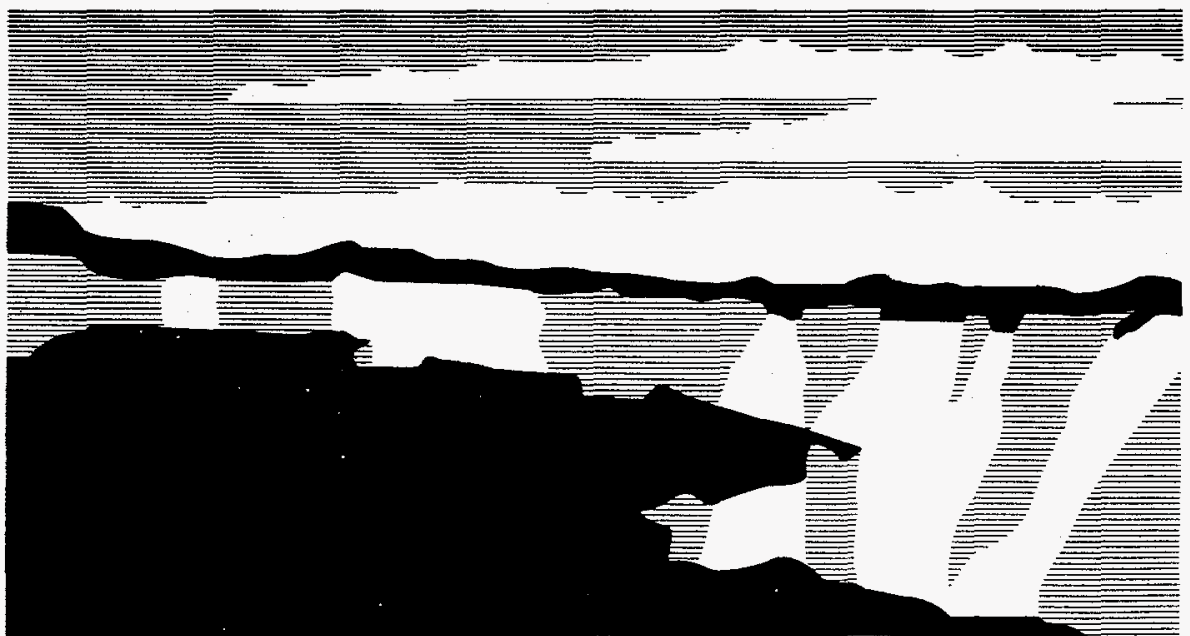

Los Alamos National Laboratory, an affirmative action/equal opportunity employer, is operated by the University of California for the U.S. Department of Energy under contract W-7405-ENG-36. By acceptance of this article, the publisher recognizes that the U.S. Government retains a nonexclusive, royalty-free license to publish or reproduce the published form of this contribution, or to allow others to do so, for U.S. Government purposes. The Los Alamos National Laboratory requests that the publisher identify this article as work performed under the auspices of the U.S. Department of Energy. 


\section{DISCLAIMER}

Portions of this document may be illegible in electronic image products. Images are produced from the best available original document. 


\title{
TIME STRUCTURE IN THE MARCH 5TH 1979 EVENT
}

\author{
E. E. Fenimore
}

Los Alamos National Laboratory, Los Alamos, New Mexico 87545, USA

\section{ABSTRACT}

The March 5th, 1979 transient occurred on a source which is also a soft gamma repeater (SGR). A new analysis of the March 5th spectrum has shown that it is similar to a classic gamma-ray burst (no soft SGR-like component). Here we show that the variation in hardness within the initial peak is similar to that found in short, classic gamma-ray bursts. An analysis of the risetime of the peak shows that the best limit that can be put on it is $\leq 1 \mathrm{~ms}$ rather than the previously reported $0.2 \mathrm{~ms}$. Classic gamma-ray bursts have shown risetimes the order of $2 \mathrm{~ms}$.

\section{INTRODUCTION}

The March 5th, 1979 gamma-ray transient is probably the most important high energy transient ever observed. March 5 th seemed to have unique features which set it aside from other events. It was dominated by an extremely intense, short peak ( $\sim 200 \mathrm{~ms}$ ) which was about 50 times brighter than any previous burst ${ }^{1}$. The peak had a very short risetime ${ }^{2}$. The spectrum of this peak was reported to be dominated by a soft component whose photons had an average energy of $30 \mathrm{keV}$ plus a hard spectral tail with $\sim 10 \%$ of the photons ${ }^{3}$. Following the bright peak were very clear pulsations with an $8 \mathrm{~s}$ period $^{1}$. In the days and months following March 5 th, there were 15 repetitions, all soft with typical photon energies of $\sim 30 \mathrm{keV}^{4}$. March 5th was seen by nine instruments resulting in a very small error box by triangulation. Within this error box is the supernova remnant (SNR) in the $\mathrm{LMC}^{5}$ known as N49.

At first, March 5th's reported soft spectrum and repetitions made it the prototype for the soft gamma repeaters (SGRs). The SGRs are events with soft spectra (average photon energies of $\sim 30 \mathrm{keV}$ ) that repeat with no clear pattern on timescales of seconds to years ${ }^{6,7}$. Recently, the SGRs have been identified $^{8,9}$ with distant SNRs implying luminosities the order of $10^{42} \mathrm{erg}$ $\mathrm{s}^{-1}$. Norris et $\mathrm{al}^{10}$ argued that March 5th should not be considered a true SGR because it was $10^{3}$ times more luminous than SGRs and the spectrum contained a hard component. March 5 th also seems very different from the classic gamma-ray bursts (GRBs). Indeed, the terminology "classic" was introduced to distinguish all other GRBs from the March 5th event. Recently, we have shown that the March 5th event might have been a classic GRB after all ${ }^{11}$. Most of its so-called unique features could have been selection effects: many GRBs could have pulsations or repetitions that were $1 \%$ of the peak intensity and they would go unnoticed. The spectrum, with its SGR-like component, was truly unique. We have shown ${ }^{11}$ that the reported spectrum $^{3}$ was probably the result of deadtime: the Konus spectrum cov- 
ered $4 \mathrm{~s}$ including $0.2 \mathrm{~s}$ of the initial peak and $3.8 \mathrm{~s}$ of the soft pulsations. However, Konus had a deadtime of $1 \mathrm{~ms}$ such that only $\sim 200$ photons came from the initial peak whereas 3800 photons came from the pulsations. A reanalysis ${ }^{11}$ of the International Comet Explorer (ICE) and Pioneer Venus Orbiter (PVO) spectral observations of March 5th revealed a spectrum that is fully consistent with a classic GRB.

\section{OBSERVATIONS OF THE MARCH 5TH PEAK}

PVO had two small CsI detectors ${ }^{12}\left(22 \mathrm{~cm}^{2}\right.$ total effective area) directly facing the south ecliptic pole near where March 5 th originated. As such, there is effectively no uncertainty in how the scintillator responds to an incident spectrum. PVO had four energy channels: 100-200, 200-500, 500-1000, and 1000-2000 keV. During non-burst times, counts are reported with a time resolution that depends on the satellite telemetry mode, often about once per second. A statistically significant variation $(11.2 \sigma)$ in the background count rate causes a special memory to record the burst with high time resolution. In this trigger mode, counts in the full energy range (100-2000 keV) are reported every $11.72 \mathrm{~ms}$ and counts in the four energy channels are reported every $187.5 \mathrm{~ms}$. For sufficiently high count rates, there is a "time-to-spill" mode where the time to record 16 counts is reported rather than the number of counts within $11.72 \mathrm{~ms}$. The March 5 th event was extremely bright such that the time-to-spill mode provided temporal resolution of $\sim 0.5 \mathrm{~ms}$ and spectral resolution with $\sim 6 \mathrm{~ms}$ resolution during the main peak.

PVO had a unusual deadtime and pulse pileup situation. The four spectral channels of PVO were defined by level discriminators but the logic gave priority to higher energy photons. This caused a coupling between pulse pileup and deadtime. Fenimore, Klebesadel, \& Laros ${ }^{11}$ describes in detail the PVO deadtime and pulse pileup effects and how we use a Monte Carlo technique to determine a best fit spectrum. To accommodate these nonlinear effects, we track individual photons through the detector response and electronics. In Fenimore, Klebesadel, \& $\operatorname{Laros}^{11}$, we found an average spectrum for the initial peak using an approximate treatment for the variations in the intensity. Here, we treat each temporal sample of the peak individually. This allows for the most accurate treatment of the deadtime and pulse pileup. We have fit two connected powers to each PVO temporal sample,

$$
\begin{array}{rr}
\phi(E)=a_{1}\left(E / E_{c}\right)^{\alpha_{1}} & E<E_{c} \\
a_{1}\left(E / E_{c}\right)^{\alpha_{2}} & E>E_{c} .
\end{array}
$$

There are four free parameters: the connecting energy $\left(E_{c}\right)$, the value of the spectrum at the connecting energy $\left(a_{1}\right)$, and the slopes above and below the connecting energy $\left(\alpha_{1}, \alpha_{2}\right)$. 
In Figure 1a, we plot the luminosity of the March 5th event as a function of time, that is,

$$
4 \pi D^{2} \int_{30}^{2000} 1.6 \times 10^{-9} E \phi(E) d E
$$

where $D$ is $55 \mathrm{kpc}$ and $\phi(E)$ is equation (1) using the best fit parameters for each sample. Here, we have chosen the Konus energy range (30 to $2000 \mathrm{keV}$ ) to facilitate comparisons to previous work. The luminosity peaks at $1.9 \times 10^{45}$ $\mathrm{erg} \mathrm{s}^{-1}$. This peak luminosity is about a factor of 4 larger than previously reported $^{3}$. Other energy ranges gives a very similar pulse shape. For the BATSE energy range (50 to $300 \mathrm{keV}$ ) the luminosity peaks at $8.7 \times 10^{44} \mathrm{erg}$ $\mathrm{s}^{-1}$. The well known features of the March 5 th time history ${ }^{2}$ are apparent: a sharp rise, followed by a gradual increase for $\sim 25 \mathrm{~ms}$, and then a decay back to background levels over $175 \mathrm{~ms}$. In Figure $1 \mathrm{~b}$ we give a hardness ratio as a function of time, defined to be

$$
H R=\frac{\int_{200}^{2000} \phi(E) d E}{\int_{100}^{200} \phi(E) d E}
$$

The variations in hardness are similar to previous reports ${ }^{13}$ although here the deadtime effects are more accurately accounted for. Also, in Fenimore et $\mathrm{al}^{13}$, there was a calibration error which led us to incorrectly conclude that the hardness ratio implied temperatures of $\sim 60 \mathrm{keV}$. The dotted line is the hardness ratio corresponding to the best fit equation (1) to the 200 ms peak ${ }^{11}$. The variations in hardness seen in March 5th are similar to that seen ${ }^{14}$ in short classical GRBs: a rough correlation of hardness and intensity with a softening at the end of the burst.

\section{MARCH 5TH RISETIME}

The rapid rise of the March 5th event is often quoted as one of its unique features. The GSFC ICE (formerly called ISEE-3) experiment and PVO were the best instruments to determine the risetime. Both the GSFC-ICE and PVO instruments had similar recording systems: they collected counts in fixed time samples $\left(\Delta T_{0}\right)$ unless the counts within $\Delta T_{0}$ exceeded some number $\left(N_{m}\right)$ in which case the number of ticks of a clock $(\Delta C)$ to obtain $N_{m}$ was reported (a time-to-spill, TTS, system). For GSFC-ICE, $N_{m}=64$ and a tick of the clock was $1 \mathrm{~ms}$. For PVO, $N_{m}=16$ and a tick of the clock was $\sim 1 / 4 \mathrm{~ms}$. For March 5th, both GSFC-ICE and PVO showed a very sharp transition from the background level to an intense plateau where $\Delta C$ was small. Let $\Delta C_{p}$ be the $\Delta C$ in the plateau $\left(\Delta C_{p}=1\right.$ for GSFC-ICE and $\Delta C_{p}=2$ for PVO). In both instruments, the transition occurred within a single TTS sample. Let $\Delta C_{t}$ be the duration of the transitional TTS sample and $\Delta C_{r}$ be the unknown time for the signal to rise to the plateau. It is possible that the risetime was instantaneous (i.e., $\Delta C_{r}=0$ ) implying that 
it occurred $\Delta C_{p}$ before the end of the transitional sample. The upper limit on the risetime is found by the constraint $N_{m}=\int_{0}^{\Delta C_{r}} f(t) d t$ where $\mathrm{f}(\mathrm{t})$ is how the bursts turns on. (Here, $t$ is in units of the ticks of the clock.) If we assume a linear turn on, $f(t)=N_{m} t /\left(\Delta C_{r} \Delta C_{p}\right)$, then $\Delta C_{r}$ is limited to $2 \Delta C_{p}$. Thus, in a TTS instrument with unresolved risetime, the linear risetime is limited to twice the duration of the TTS samples after the rise. For the case of March 5th, GSFC-ICE limits the rise to $2 \mathrm{~ms}$ and PVO limits it to $1 \mathrm{~ms}$ (see ref. 15).

Our limit on the risetime from GSFC-ICE $(2 \mathrm{~ms})$ is larger than the often quoted ${ }^{2}$ limit of $0.2 \mathrm{~ms}$. However, $0.2 \mathrm{~ms}$ is not a risetime but a time constant to increase the signal two orders of magnitude in less than $1 \mathrm{~ms}$. Such a time constant is not a measure of the risetime; note that it depends on the ratio of the peak to the background.

Other bursts have comparable risetimes. For example, GB841215 ${ }^{16}$ and GB900711 17 had risetimes the order of 2 to $3 \mathrm{~ms}$ and GB900711 shows significant structure at $0.2 \mathrm{~ms}$. Since the risetime in a TTS system is $2 \Delta C_{p}$ and $\Delta C_{p}$ is inversely proportional to the peak count rate, if March 5 th was, say, a factor of 5 times weaker, the upper limit on its risetime would be $\sim 5$ ms. We conclude that the risetime of March 5 th is not a unique feature that distinguishes it from other GRBs.

\section{REFERENCES}

1. E. P. Mazets et al. 1979 Nature 282,587

2. T. L. Cline et al 1980 ApJ 237 L1

3. E. P. Mazets 1982 Astrophys. Space Sci., 84173

4. S. V. Golenetskii, V. N. Ilyinskii, \& E. P. Mazets 1984 Nature 307,41

5. W. D. Evans et al 1980 ApJ 237 L7

6. J. G. Laros et al 1987 ApJ 320, L111

7. J.-L. Atteia et al 1987 ApJ 320, L105

8. S. R. Kulkarni \& D. A. Frail 1993 Nature 365, 33

9. K. Hurley et al 1994 ApJ 431, L31

10. J. P. Norris et al 1991 ApJ 366, 240

11. E. E. Fenimore, R. W. Klebesadel, \& J. G. Laros ApJ submitted

12. R. W. Klebesadel et al 1980 IEEE Trans. Geosic. Electron., GE-18 76

13. E. E. Fenimore et al. 1981 Nature 289,42

14. J. P. Norris et al. 1995, ApJ submitted

15. J. Terrell, et al. 1980 Nature 285383

16. J. G. Laros et al 1985 Nature 318,448

17. P. N. Bhat et al 1992 Nature 359, 217 
Figure 1. (a) The luminosity structure of the peak of the March 5th event. Each sample has been corrected for deadtime and pulse pileup. The Konus energy range of 30 to $2000 \mathrm{keV}$ was used and a distance of $55 \mathrm{kpc}$ was assumed. The risetime is $\leq 1 \mathrm{~ms}$. (b) Hardness ratio as a function of time for the March 5th event. The ratio was defined as the photons between 200 and $2000 \mathrm{keV}$ compared to those between 100 and $200 \mathrm{keV}$. The hardness evolution is similar to that seen in classic gamma-ray bursts. The dashed line is the corresponding hardness for an average over the peak ${ }^{11}$. 


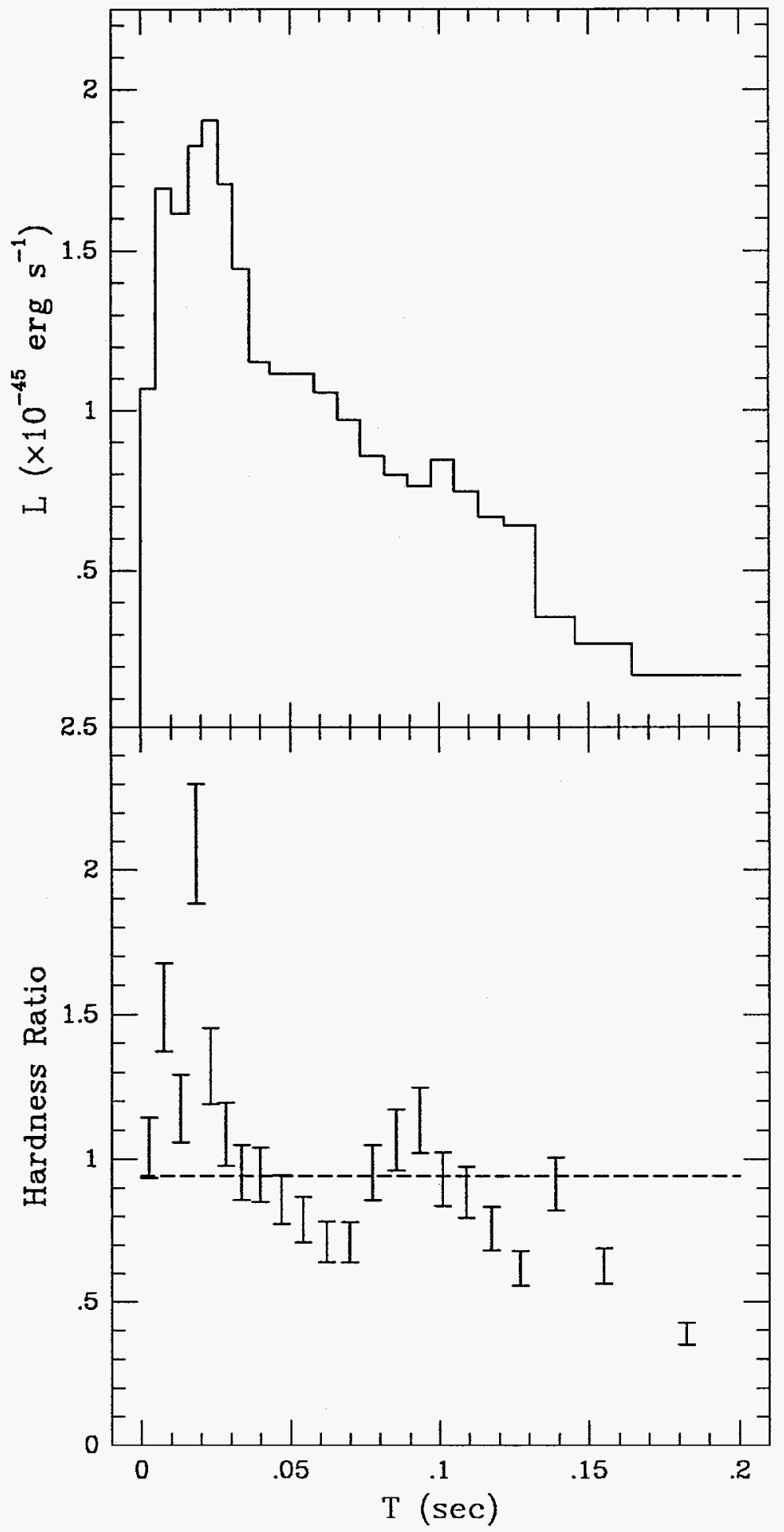

